\title{
Metastatic Thyroid Follicular Carcinoma Presenting as a Primary Renal Tumor
}

\author{
Huan $\mathrm{Xu}^{1}$, Wentong Zeng ${ }^{2}$ and Ying Tang ${ }^{1}$
}

\begin{abstract}
Renal tumor metastasis from thyroid follicular carcinoma is very rare. Here, we present a 64-year-old woman with metastatic thyroid follicular carcinoma presenting as a primary renal tumor. The renal tumor was removed and revealed the origin of thyroid follicular carcinoma. Six months after the treatment of renal tumor, the patient was readmitted for the enlarged thyroid gland. The follicular carcinoma was revealed in the resected thyroid gland. The serum thyroglobulin level and thyroid function tests were consistently normal. Although with metastasis to the kidney, thyroid follicular carcinoma is thought to have a good prognosis.
\end{abstract}

Key words: thyroid follicular carcinoma, renal metastasis

(Intern Med 51: 2193-2196, 2012)

(DOI: 10.2169/internalmedicine.51.7495)

\section{Introduction}

Follicular carcinoma of the thyroid accounts for about 10$15 \%$ of thyroid malignancy. It mainly affects elderly females. Follicular carcinoma mainly spreads hematogenously. At the time of diagnosis, distant metastasis was found in more than $20 \%$ of cases, in particular metastasis to the lungs and bones (1). The rare sites of distant metastasis are the brain, skin, soft tissue, liver, and so on (2). Metastatic thyroid follicular carcinoma presenting as a primary renal tumor is especially rare. Here we report a case of renal metastasis from thyroid follicular carcinoma.

\section{Case Report}

A 64-year-old woman complained of flank dull pain for 1 week without hematuria. The abdominal computed tomography (CT) revealed a $2.8 \mathrm{~cm} \times 2.3 \mathrm{~cm}$ mass on the left kidney (Fig. 1), considered as renal cell carcinoma. She was admitted to our hospital for treatment of the renal tumor in July 2010.

Physical examinations on admission showed: body temperature $36.5^{\circ} \mathrm{C}$, pulse rate 82 beats $/ \mathrm{min}$, blood pressure $125 / 86 \mathrm{mmHg}$, respiratory rate $21 \mathrm{counts} / \mathrm{min}$. There was no palpable enlarged lymph node on her cervical, axillary or inguinal area, and no palpable mass on her abdominal region.

Laboratory data on admission showed microhematuria at urinalysis without protein, glucose and white blood cell. The blood cell count and biochemical data were normal. The examinations of blood thyroglobulin ( $\mathrm{Tg}$ ) level and antithyroglobulin antibody were not done. Chest X-ray revealed no lesion on the lung or heart.

The patient underwent left nephrectomy 6 days after her admission. The resected kidney measured $10 \mathrm{~cm} \times 6 \mathrm{~cm} \times 5$ $\mathrm{cm}$. The tumor located on the middle part of the kidney measured $2.5 \mathrm{~cm} \times 2 \mathrm{~cm} \times 2 \mathrm{~cm}$, protruding to the capsule. By histological examination, the tumor cells were arranged in a well-differentiated follicular pattern. Colloid-like materials were found in the intrafollicular space (Fig. 2). The strong positive immunohistochemical stain of thyroglobulin (TG) and thyroid transcription factor-1 (TTF-1) revealed the origin of thyroid gland (Fig. 3). The patient was discharged without receiving the pathology report. We informed her of the final diagnosis and advised her to return for an examination of the thyroid gland. But she refused.

Six months after her nephrectomy, she was readmitted to our hospital for an enlarged thyroid in February 2011. A palpable non-tender mass was identified in the right lobe of the thyroid gland during the physical examination. The cer-

${ }^{1}$ Department of Pathology, The West China Hospital of Sichuan University, China and ${ }^{2}$ Department of Urology, The Affiliated Hospital of Chengdu University of Traditional Chinese Medicine, China

Received for publication February 8, 2012; Accepted for publication March 11, 2012

Correspondence to Dr. Huan Xu, xu141@ sohu.com 


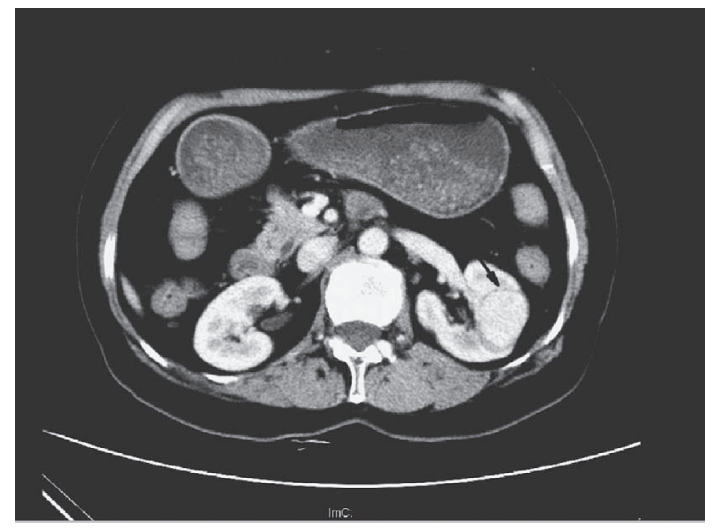

Figure 1. Abdominal CT revealed a $2.8 \mathrm{~cm} \times 2.3 \mathrm{~cm}$ mass on the left kidney (arrow).

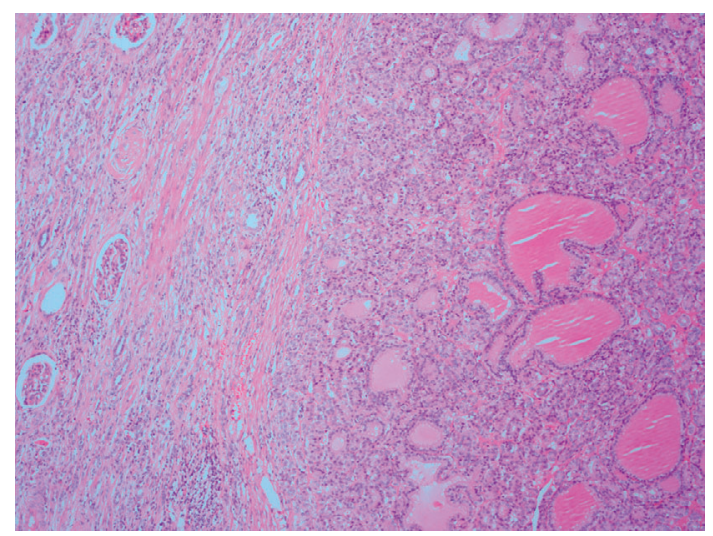

Figure 2. Histological findings revealed the tumor cells were arranged in well differentiated follicular pattern. Colloid-like materials were found in the intrafollicular space. (Hematoxylin and Eosin staining: original magnification $\times 40$ ).

vical CT scan revealed multiple nodules in the enlarged thyroid gland. The largest one located on the right lobe measured $3.9 \mathrm{~cm} \times 2.9 \mathrm{~cm}$ with dot and ring calcification in the nodule (Fig. 4). No enlarged lymph node was found in the neck region. Blood cell count, serum calcium and phosphate levels, and thyroid function tests were all normal. The serum TG level was $52.48 \mathrm{ng} / \mathrm{mL}$ and the anti-thyroglobulin antibody was $15.89 \mathrm{IU} / \mathrm{mL}$. Then total thyroidectomy with a lymphadenectomy was performed. Histological examination revealed the well-differentiated follicular carcinoma with extensive capsular and vascular invasion in the right lobe of the thyroid and the nodular goiter in the residual tissues (Fig. 5). All the resected lymph nodes were spared of tumor cells.

The patient was discharged in February 2011. She received levothyroxine replacement therapy at a daily dose of $75 \mu \mathrm{g}$. She is still in good health without recurring lesion at the latest follow-up in February 2012.

\section{Discussion}

Follicular carcinoma is the second most common differen- tiated thyroid malignancy. It mainly affects elderly females, especially those over 50 years old. This tumor is often seen in patients with a long-standing non-toxic nodular goiter history. We also found a change of nodular goiter in the present patient. Well-differentiated follicular carcinoma is difficult to differentiate from follicular adenoma and histological evidence of capsular or vascular invasion is necessary. These distant metastases may occur at any time from 0 to 37 years after the diagnosis of follicular carcinoma $(3,4)$. Distant metastasis to the kidney is very rare. Only 18 cases of renal metastasis from thyroid carcinoma have been reported in the English language literature, and 4 cases were first regarded as the primary renal tumor. The renal metastatic thyroid carcinomas are mainly well-differentiated follicular carcinoma and papillary carcinoma. There are few cases of Hurthle cell thyroid carcinoma and poorly differentiated carcinoma (5-8). But there have been more than 30 cases of renal metastasis of thyroid carcinoma reported in the Japanese language literature, which may indicate that the incidence of thyroid carcinoma is higher in Japan.

Several reports have suggested that the results of radiographic imaging examinations, such as fluorodeoxyglucose positron emission tomography-computed tomography, magnetic resonance imaging and Somatostatin receptor scintigraphy with single photon emission computed tomography (SPECT)-computed tomography, are helpful for the diagnosis of metastatic renal carcinoma of thyroid origin $(8,9)$. However, the present patient complained of symptoms of the kidney first, and she only underwent abdominal computed tomography before her first surgery of kidney. Because the renal mass was single, without satellite foci, and metastatic lesions are mostly multiple, primary renal cell carcinoma was considered by the radiographic examination. It is helpful to make the differential diagnosis by taking a variety of multimodality imaging techniques before the surgery.

The differentiation between the renal metastasis of thyroid follicular carcinoma and the thyroid follicular carcinoma-like renal tumor is very important. As we know, renal cell carcinomas are characterized by different histological patterns and clinical courses. Thyroid follicular carcinoma-like renal tumor, which morphologically resembles that of thyroid follicular carcinoma, is a new histological pattern of primary renal tumor. It was first reported in 2006 (10). Immunohistochemistry plays an important role in the differential diagnosis. The tumor cells of renal cell carcinoma are stained positively for cytokeratin (CK7, CK20, CAM 5.2), vimentin, CD10 and CD117, but TTF-1 and TG are stained completely negative. The positive stain of TTF-1 and TG are sensitive and specific for the primary and metastatic thyroid tumor (11).

Generally an elevated serum $\mathrm{Tg}$ level is associated with differentiated thyroid carcinoma. In the present patient, the blood TG level was in the normal range and there was no symptom of thyroid dysfunction. It has been reported that the serum Tg level of 127 cases of thyroid follicular carcinoma ranged from 2 to $18,640 \mathrm{ng} / \mathrm{mL}$ (12). Thus, we cannot 

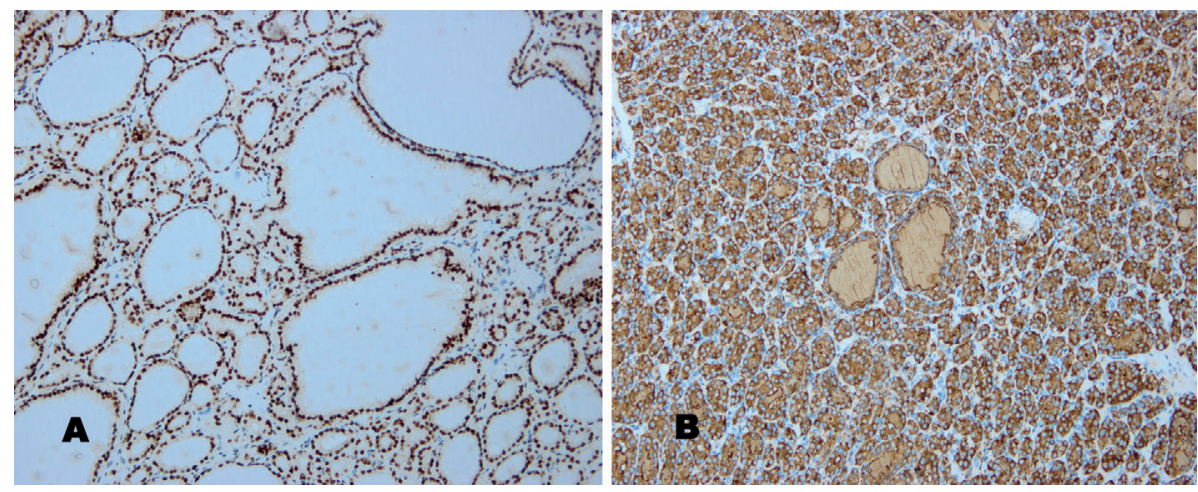

Figure 3. The strong positive immunohistochemical stain of thyroid transcription factor-1(A) and thyroglobulin(B). (Immunohistochemistry stain: original magnification $\times \mathbf{1 0 0}$ ).

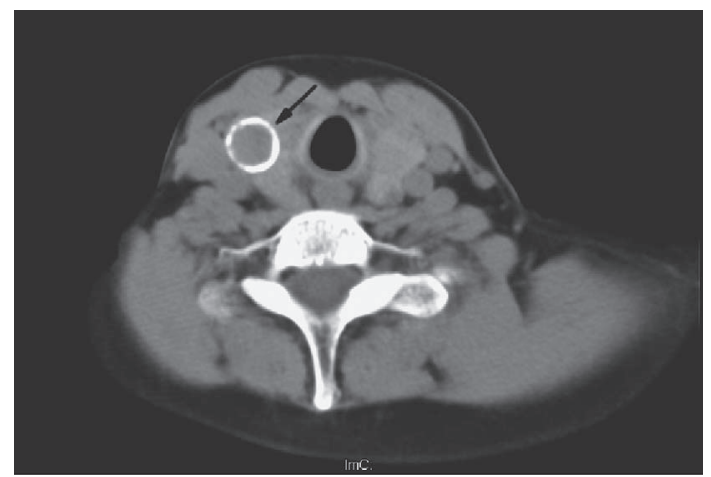

Figure 4. Cervical CT revealed multiple nodules in the enlarged thyroid gland. The largest one located in the right lobe measured $3.9 \mathrm{~cm} \times 2.9 \mathrm{~cm}$ with dot and ring calcification in the nodule (arrow).

rule out the diagnosis of follicular carcinoma if the serum TG level is not so high. But serum Tg level is still a useful indicator for the monitoring of thyroid tumor recurrence or metastasis (13).

The recommend that the treatment for the welldifferentiated follicular carcinoma with distant metastasis is maximal excision of the tumors followed by thyroid hormone supplementation and iodine-131 therapy. This patient only received thyroid hormone supplementation after the total resection of the tumors and she is in good health at the last follow-up. The maximal excision of the tumors plays the most important role in the good prognosis of thyroid carcinoma.

This is a rare case of thyroid follicular carcinoma which metastasized to the kidney as a primary tumor. The follicular pattern and the strong immunostaining of TG and TTF-1 are useful to make the correct histological diagnosis. Although it is unusual, metastatic tumor of the kidney may originate from the thyroid gland. Complete surgical excision of the distant metastatic lesion and primary tumor can still offer a good prognosis.

The authors state that they have no Conflict of Interest (COI).

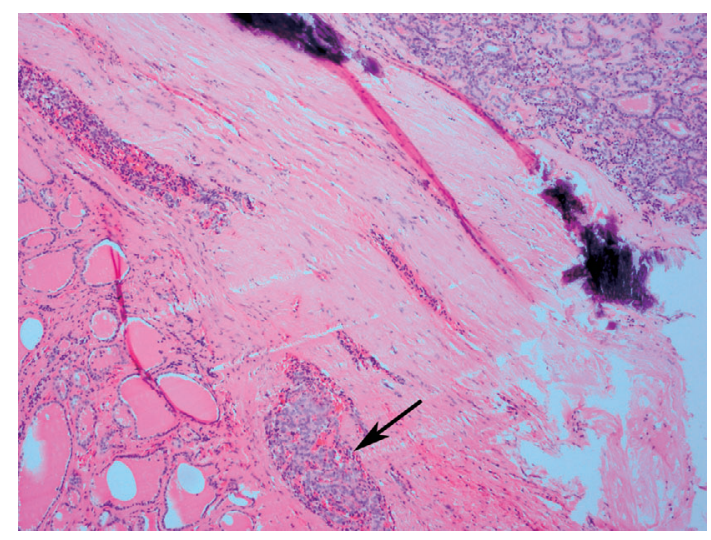

Figure 5. Histological findings revealed the follicular carcinoma of the thyroid. The black arrow refers to the vascular invasion. (Hematoxylin and Eosin staining: original magnification $\times \mathbf{4 0})$.

\section{References}

1. Emerick GT, Duh QY, Siperstein AE, et al. Diagnosis, treatment, and outcome of follicular thyroid carcinoma. Cancer 72: 32873295, 1993.

2. Song HJ, Xue YL, Xu YH, et al. Rare metastases of differentiated thyroid carcinoma: pictorial review. Endocr Relat Cancer 18: R165-R174, 2011.

3. Johnson MW, Morettin LB, Sarles HE, Zaharopoulos P. Follicular carcinoma of the thyroid metastatic to the kidney 37 years after resection of the primary tumor. J Urol 127: 114-116, 1982.

4. Gamboa-Dominguez A, Tenorio-Villalvazo A. Metastatic follicular variant of papillary thyroid carcinoma manifested as a primary renal neoplasm. Endocr Pathol 10: 256-268, 1999.

5. Iwai H, Ohno Y, Ito H, Kiyokawa T, Aoki N. Renal rupture associated with a poorly differentiated follicular thyroid carcinoma metastasizing to the thigh muscle, lung and kidney. Intern Med 44: 848-852, 2005

6. Moudouni SM, En-Nia I, Rioux-Leclerq N, et al. Follicular carcinoma of the thyroid metastasis to the kidney nine years after resection of the primary tumor. Ann Urol 36: 36-37, 2002.

7. Liou MJ, Lin JD, Chung MH, et al. Renal metastasis from papillary thyroid microcarcinoma. Acta Otolaryngol 125: 438-444, 2005.

8. Djekidel M, Gordon M, Shah RB, et al. Renal metastasis from Hurthle cell thyroid carcinoma and its evaluation with hybrid im- 
aging. Thyroid 20: 429-433, 2010.

9. Shiga T, Tsukamoto E, Nakada K, et al. Comparison of (18)FFDG, (131)I-Na, and (201)Tl in diagnosis of recurrent or metastatic thyroid carcinoma. J Nucl Med 42: 414-419, 2001.

10. Jung SJ, Chung JI, Park SH, et al. Thyroid follicular carcinomalike tumor of kidney: a case report with morphologic, immunohistochemical, and genetic analysis. Am J Surg Pathol 30: 411-415, 2006.

11. Cimino-Mathews A, Sharma R, Netto GJ. Diagnostic use of PAX 8, CAIX, TTF-1, and TGB in metastatic renal cell carcinoma of the thyroid. Am J Surg Pathol 35: 757-761, 2011.

12. Petric R, Perhavec A, Gazic B, Besic N. Preoperative serum thyroglobulin concentration is an independent predictive factor of malignancy in follicular neoplasms of the thyroid gland. J Surg Oncol 105: 351-356, 2012.

13. Pelttari H, Välimäki MJ, Löyttyniemi E, Schalin-Jäntti C. Postablative serum thyroglobulin is an independent predictor of recurrence in low-risk differentiated thyroid carcinoma: a 16-year follow-up study. Eur J Endocrinol 163: 757-763, 2010.

(C) 2012 The Japanese Society of Internal Medicine http://www.naika.or.jp/imonline/index.html 